\title{
Risk factors for ulceration and amputation in patients with diabetic foot at risk: results form a tertiary care center
}

\author{
Adriana Russo Fiore*, Arnaldo Moura Neto, Karla Borges Daniel, Walter José Minicucci, \\ Denise Engelbrecht Zantut Wittmann, Marcos Antonio Tambascia, Elizabeth João Pavin, \\ Maria Cândida Ribeiro Parisi
}

From 20th Brazilian Diabetes Society Congress

Porto Alegre, Brazil. 11-18 November 2015

\section{Background}

Ulceration and amputation are severe complications of diabetes, leading to great morbidity and mortality. Of all lower limb amputations, about $50 \%$ are performed in these patients (1). In their lifetime, diabetics have a chance as high as $25 \%$ to develop a foot ulcer (2). After an amputation, mortality rates ranges from $13 \%$ to $40 \%$ at 1 year, $35 \%$ to $65 \%$ at 3 yrs., and $39 \%$ to $80 \%$ at 5 yrs. (3). The aim of this study was to assess the main risk factors of ulceration and amputation in patients with type 1 and type 2 diabetes.

\section{Materials and methods}

A cross-sectional study was conducted in a tertiary hospital. Data was collected on the patients' first attendance in the neuropathic and diabetic foot unit, between June 2012 and September 2014. Statistical significance was set at 5\%.

\section{Results}

A total of 177 patients were evaluated. Ulceration and amputation were significantly more frequent in men (70.2\% of all ulcerated patients and $76.9 \%$ of all amputees; $\mathrm{p}<0.001)$. Hypertension was also a risk factor for amputation, present in $74.5 \%$ of amputated patients $(\mathrm{p}=0.034)$. In ulcerated patients, glycated hemoglobin was higher than in non-ulcerated patients $(9.2 \pm 2 \%$ vs $8.35 \pm 1.99 \% ; \mathrm{p}=0.003)$. The independent risk factors for ulceration and amputation were neuropathic and neuroischemic foot at risk classification (OR 4.41; CI 1.83 10.65; p 0.001 and OR 1.21; CI $2.07-60.47$; p =0.005, respectively), dyslipidemia increased (OR 9.2; CI 1.64 51.58; $\mathrm{p}=0.012$ and OR 5.68; CI $1.21-26.46$; $\mathrm{p}=0.027$, respectively) and microalbuminuria (OR 1.004; CI 1.001 - 1.006; $\mathrm{p}=0.011$ and OR 1.005; CI $1.001-1.008$; $\mathrm{p}<$ 0.001 , respectively). There were no statistically significant differences between risk of ulcer or amputation and ethnicity, age, type of diabetes, duration of diabetes, BMI (body mass index), Neuropathic Symptom Score, Neuropathy Disability Score, heart disease and retinopathy (Figure 1).

\section{Conclusion}

Besides neuropathic and neuro-ischemic foot, other factors such as hypertension, dyslipidemia and presence of microalbuminuria were variables associated with ulceration and amputation, supporting that treatment of the diabetic patient should also aim the control of cholesterol levels and microalbuminuria. Specific care and education should be directed to males, due worse outcomes. The knowledge of these risk factors is important for implementation of prevention strategies, avoiding future damage and disability.

Published: 11 November 2015

\section{doi:10.1186/1758-5996-7-S1-A18}

Cite this article as: Fiore et al:: Risk factors for ulceration and

amputation in patients with diabetic foot at risk: results form a tertiary care center. Diabetology \& Metabolic Syndrome 2015 7(Suppl 1):A18.

* Correspondence: adriana.carneiro@gmail.com 


\begin{tabular}{|c|c|c|c|c|c|c|}
\hline \multirow[b]{2}{*}{ Variable } & \multicolumn{2}{|c|}{ Ulceration } & \multirow[t]{2}{*}{$p$} & \multicolumn{2}{|c|}{ Amputation } & \multirow[t]{2}{*}{$p$} \\
\hline & Present & Absent & & Present & Absent & \\
\hline Gender (\%) & & & $<0.001$ & & & $<0.001$ \\
\hline Female & 29.8 & 62.7 & & 23.1 & 52.9 & \\
\hline Male & 70.2 & 37.3 & & 76.9 & 47.1 & \\
\hline Ethnicity (\%) & & & 0.159 & & & 0.065 \\
\hline White & 75.8 & 65.3 & & 78.4 & 67.5 & \\
\hline Black & 8.8 & 18.7 & & 15.7 & 12.3 & \\
\hline Brown & 15.4 & 16 & & 5.9 & 20.2 & \\
\hline Age (mean \pm SD)(years) & $58.38 \pm 12.6$ & $57.47 \pm 14.7$ & 0.798 & $60.45 \pm 10.7$ & $57.33 \pm 14.6$ & 0.147 \\
\hline Type of diabetes (\%) & & & 0.234 & & & 0.811 \\
\hline $\begin{array}{ll}\text { Type } 1 \text { diabetes } \\
\end{array}$ & 12.8 & 7.3 & & 9.6 & 10.8 & \\
\hline Type 2 diabetes & 87.2 & 92.7 & & 90.4 & 89.2 & \\
\hline $\begin{array}{l}\text { Duration of diabetes } \\
\text { (years) (mean } \pm \mathrm{SD} \text { ) }\end{array}$ & $17.2 \pm 10$ & $13.2 \pm 9.4$ & 0.408 & $17.3 \pm 10.4$ & $13.6 \pm 9.6$ & 0.239 \\
\hline BMS $\left(\mathrm{kg} / \mathrm{m}^{2}\right)($ mean $\pm S D)$ & $29.4 \pm 6.1$ & $29.3 \pm 6.2$ & 0.061 & $28.7 \pm 5.5$ & $29.4 \pm 6.2$ & 0.069 \\
\hline Neuropathy Symptom Score (\%) & & & 0.794 & & & 0.416 \\
\hline Normal or mild & 56.7 & 59 & & 51.4 & 59.5 & \\
\hline Moderate or severe & 43.3 & 41 & & 48.6 & 40.5 & \\
\hline Neuropathy Disability Score (\%) & & & 0.670 & & & 0.883 \\
\hline Normal or mild & 51.9 & 55.4 & & 56.4 & 55 & \\
\hline Moderate or severe & 48.1 & 44.6 & & 43.6 & 45 & \\
\hline Heart disease (\%) & & & 0.090 & & & 0.320 \\
\hline Present & 33.3 & 49 & & 33.3 & 43.2 & \\
\hline Absent & 66.7 & 51 & & 66.7 & 56.8 & \\
\hline Dyslipidemia (\%) & & & 0.156 & & & 0.227 \\
\hline Present & 69.6 & 79.5 & & 68.2 & 77.6 & \\
\hline Absent & 30.4 & 20.5 & & 31.8 & 22.4 & \\
\hline Hypertension (\%) & & & 0.776 & & & 0.034 \\
\hline Present & 85.9 & 84.3 & & 74.5 & 87.6 & \\
\hline Absent & 14.1 & 15.7 & & 25.5 & 12.4 & \\
\hline Retinopathy (\%) & & & 0.771 & & & 0.501 \\
\hline Present & 77.5 & 75.4 & & 72.5 & 77.9 & \\
\hline Absent & 22.5 & 24.6 & & 27.5 & 22.1 & \\
\hline Type of feet (\%) & & & 0.182 & & & 0.345 \\
\hline Normal & 1.1 & 0 & & 1.9 & 0 & \\
\hline Neuropathic & 59.6 & 60 & & 50 & 60.2 & \\
\hline $\begin{aligned} \text { Ischemic } \\
\end{aligned}$ & 1.1 & 7.1 & & 3.8 & 3.7 & \\
\hline Both (neuropathic and ischemic) & 38.2 & 32.9 & & 44.2 & 36.1 & \\
\hline
\end{tabular}

Figure 1 Associated factors with ulceration and amputation - description of the population (SD, standard deviation) 